\title{
Magnetization Processes of Hard Magnetic Composites - Monte Carlo Studies
}

\author{
G. ZióŁkowski*, K. Granek and A. Chrobak \\ Institute of Physics, University of Silesia, Uniwersytecka 4, 40-007 Katowice, Poland
}

\begin{abstract}
In the present work we performed some simulated annealing plus Monte Carlo studies concerning a hard magnetic spherical particles embedded into soft ferromagnetic matrix as well as irregular branches of soft and hard magnetic phases. Magnetization process of such system depends on exchange interactions of soft and hard magnetic object and interface between them. Especially interesting is the comparison of interactions in systems with different, regular and irregular geometry.
\end{abstract}

DOI: 10.12693/APhysPolA.133.663

PACS/topics: $75.50 . \mathrm{Ww}, 75.60 . \mathrm{Ej}, 02.70 .-\mathrm{c}$

\section{Introduction}

Magnetic materials are very important in nowadays technologies [1-3]. New and continuously increasing requirements can be fulfilled by modern nanostructured magnetic composites containing phases characterized by different magnetic properties. Recently, we reported ultra-high coercivity $(>7 \mathrm{~T})$ in Fe-Nb-B-Tb type of bulk nanocrystalline alloys prepared by the vacuum suction technique [4-6]. It was shown that in such materials the interactions between relatively soft $\left(\mathrm{TbFe}_{2}\right)$ and hard magnetic phases $\left(\mathrm{Tb}_{2} \mathrm{Fe}_{14} \mathrm{~B}\right)$ with specific irregular branches are especially important and can lead to an appearing of new and unique properties. On the other hand, the antiferromagnetic coupling between $\mathrm{Fe}$ and $\mathrm{Tb}$ limits the maximum of magnetic saturation and, as a consequence, reduces the application potential for such materials. However, the grains with ultra-high coercivity may be a key element for the composites containing also some soft magnetic phases (like $\mathrm{Fe}$ ) and via the interactions with their surrounding can lead to improvement of the composite magnetic remanence. Therefore, a better understanding the interactions in such systems, including optimal balance between soft and hard phases is especially interesting.

The aim of this paper is to study magnetization processes of a system containing magnetic moments with and whithout magnetic anisotropy (magnetically hard and soft phases) in two defined geometries: i) a ball in soft magnetic matrix and ii) irregular based on the Perlin noise function.

\section{Simulation procedure and system}

In the present work we performed simulated annealing plus Monte Carlo studies concerning a mixture of magnetically soft and hard phases. The algorithm details are

\footnotetext{
*corresponding author; e-mail: gziolkowski@us.edu.pl
}

described in [7]. The simulations were performed for the three kinds of systems:

1. a spherical hard magnetic particle, inside nonmagnetic surroundings (typical Stoner-Wohlfarth particle),

2. a hard magnetic spherical particles embedded into soft ferromagnetic matrix (see Fig. 1a),

3. soft ferromagnetic matrix consists, irregular branches of hard magnetic phase, generated by the so-called 3D Perlin noise procedure [8], which gives statistical noise with a mean value equal to zero in each direction (composite structure, see Fig. 1b).

Moreover, the hard and soft regions are separated by the transition area with random anisotropy axis corresponds to the grain boundary in the real system.

Each structure was simulated three times with proportions of 30,50 and $70 \%$ between the hard phase volume and the whole system, with $N=50^{3}$ magnetic moments.

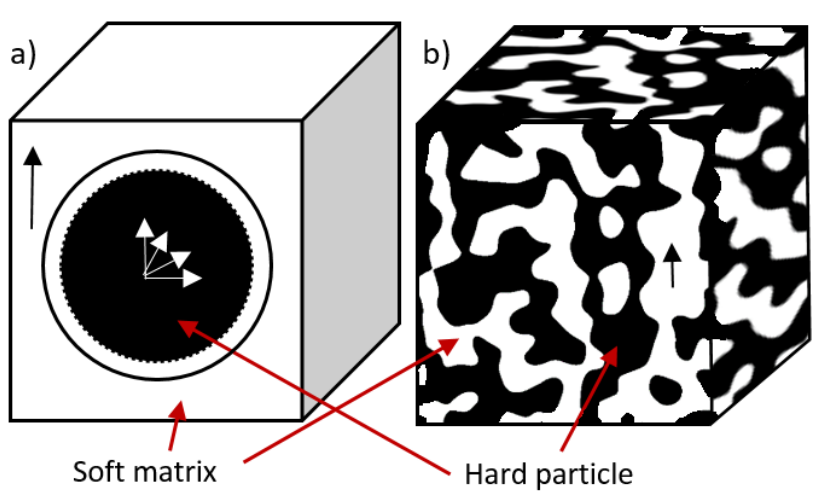

Fig. 1. Demonstration of two simulated systems: a) spherical particle and b) irregular branches generated by Perlin noise.

Energy of the system was calculated in the frame of the 3D Heisenberg model: 


$$
\begin{aligned}
E & =-\sum_{i, j} J_{i, j} \boldsymbol{S}_{i} \boldsymbol{S}_{j}-\sum_{i} K_{i}\left(\boldsymbol{S}_{i} \cdot \boldsymbol{n}_{i}\right)^{2} \\
& -g \mu_{\mathrm{B}} \mu_{0} \sum_{i} \boldsymbol{H}_{i} \cdot \boldsymbol{S}_{i},
\end{aligned}
$$

where: $J_{i, j}$ is the exchange parameter, $\boldsymbol{S}_{i}$ is the spin vector on site $i, K_{i}$ is the anisotropy constant (per site), $\boldsymbol{n}_{i}$ is the easy magnetization axis, $g$ is the Lande factor, $\mu_{\mathrm{B}}$ is the Bohr magneton, $\mu_{0}$ is the vacuum permeability and $\boldsymbol{H}_{i}$ is the magnetic field on site $i$. Note, that the dipolar interaction are neglected due to the high anisotropy. In the frame of the presented simulations the main parameters are equal to: $S=1, K=0$ and $10^{-3}$ as well as $J=1.4^{-2}$ and $0.93^{-2}$ for the soft and hard regions, respectively. The exchange parameters corresponds to the Curie temperature of about $1000 \mathrm{~K}$ and $650 \mathrm{~K}$.

The simulation process has three main steps:

1. Slow cooling of the system to the $k_{\mathrm{B}} T=10^{-4}$, based on the Simulated Annealing procedure.

2. Sequence of magnetic field changes according to Monte Carlo procedure including $5 \times 10^{8}$ iterations per field change and cluster analysis.

3. The average hysteresis loop is the average of 9 simulations performed for 9 angles from 0 to 90 degrees between easy magnetization axis and external magnetic field direction ( $z$ axis) for the hard magnetic component (in this paper labeled $\theta$ ).

Finally, the preformed simulations consist about 10000 core hours of calculations on the $2.4 \mathrm{GHz}$ units.

\section{Results and discussion}

Figure 2 presents the magnetic hysteresis loops obtained for system with spherical hard magnetic particle (50 vol.\%) embedded into non-magnetic surrounding. This case relates to the Stoner-Wohlfarth (S-W) model of magnetization of superparamagnetic particles. As might be expected, for angle between easy magnetization axis and magnetic field equal to $90^{\circ}$, a straight line was observed. On the other hand the rectangular hysteresis with the highest anisotropy field (17 T) corresponds to $\theta=0^{\circ}$. In the case of real systems one may assume that the material collect many grains with different anisotropy directions. Therefore, the average curve, as a superposition of each angles, should be analyzed. Note, that the deflection of average hysteresis about $\pm 12 \mathrm{~T}$ of external magnetic field has no physical meaning and it is a result of small number of curves to averaging around $\theta=0^{\circ}$. The coercivity of the average hysteresis is equal to $8.8 \mathrm{~T}$ and it's independent on the volume of the particle.

Interesting is the comparison of above results with the system including soft ferromagnetic matrix (see Fig. 3). In this case, the key meaning has coupling between hard particle and soft surrounding as well as balance between magnetocrystalline energy of soft matrix and high anisotropy energy of the particle. One can notice that,

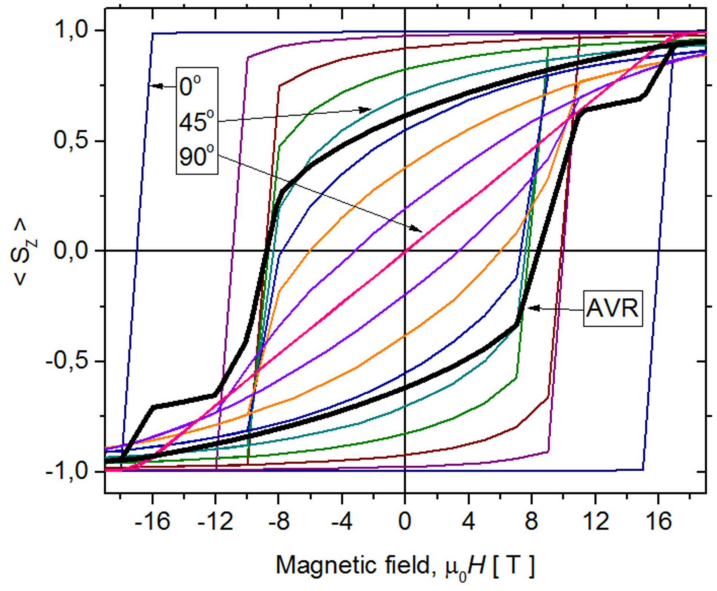

Fig. 2. The simulations of spherical hard magnetic particle (50 vol.\%) embedded into non-magnetic surrounding for $\theta=0^{\circ} \div-90^{\circ}$.

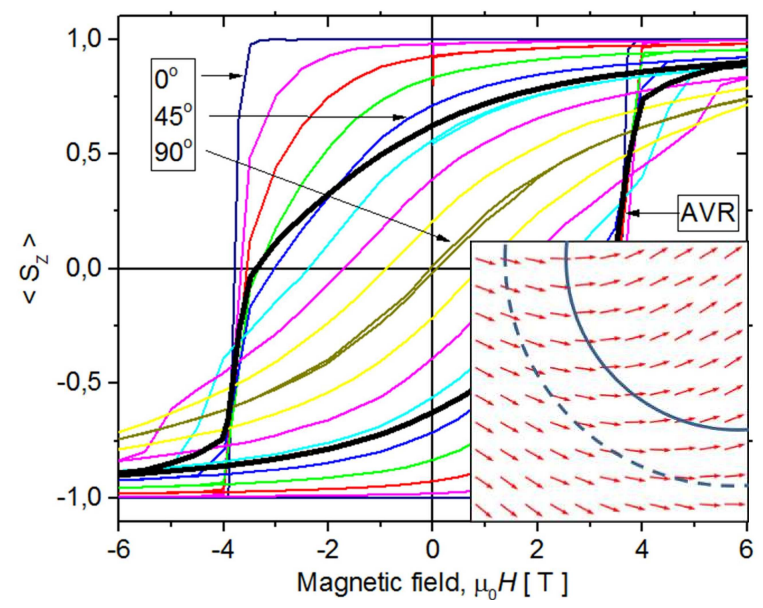

Fig. 3. The simulations of spherical hard magnetic particle (50 vol.\%) embedded into ferromagnetic surrounding for $\theta=0^{\circ} \div 90^{\circ}$ and a part of magnetic moments system presented in inset.

the shape of average hysteresis loop is typical for single phase or coupled systems and the coercivity is much lower than in the case of free particle (equals $3.4 \mathrm{~T}$ for $50 \mathrm{vol} . \%$ ) as well as increases from $1.35 \mathrm{~T}$ up to $4.8 \mathrm{~T}$ for $30 \%$ and $70 \%$ of the particle volume, respectively. The inset of Fig. 3. presents a small part of magnetic moments system from the cross section for selected point of simulation (-3.5 T, close to coercivity). Moreover, the particle sphere (solid line) and boundary region (dot line) were also marked.

As we mentioned before, in the case of high coercive materials like $\mathrm{Fe}-\mathrm{Nb}-\mathrm{B}-\mathrm{Tb}$ alloys prepared by the vacuum suction technique, very important meaning has a specific irregular microstructure [6]. Therefore, especially interesting is the comparison of the two systems with regular and irregular geometry. The disordered structure was generated by the Perlin noise procedure with periodic boundary conditions. The obtained magnetic hysteresis 


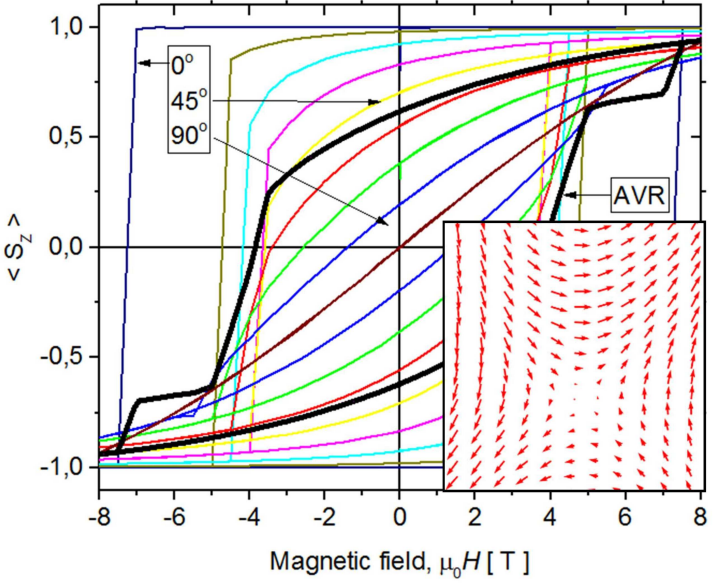

Fig. 4. The simulations of the Perlin noise system (50 vol.\%) for $\theta=0^{\circ} \div 90^{\circ}$ and a part of magnetic moments system presented in inset.

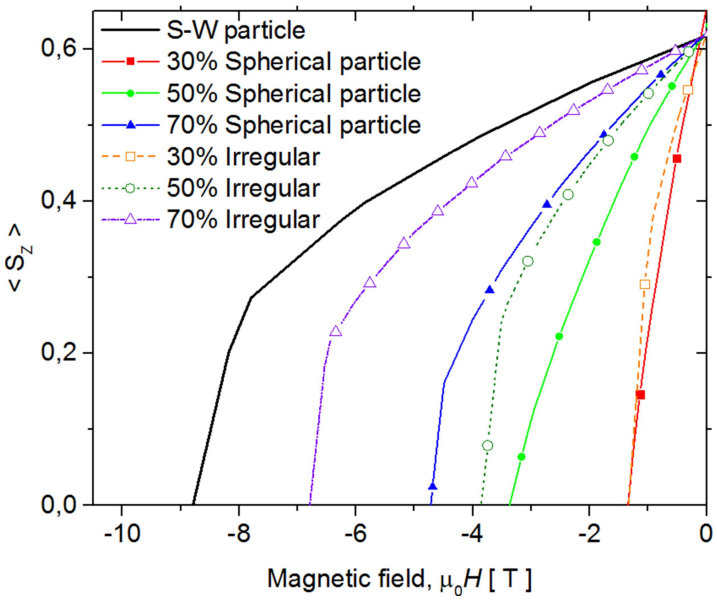

Fig. 5. The comparison of average hysteresis loops for $\mathrm{S}-\mathrm{W}$ particle, as well as regular and irregular system.

loops for 50 vol.\% of hard particle is presented in Fig. 4. Note, that the anisotropy field (for $\theta=0^{\circ}$ ), exceeds $7 \mathrm{~T}$ in contrast to less than $4 \mathrm{~T}$ for the spherical particle. This difference increases with increasing volume of the hard phase. In case of 70 vol. \% the anisotropy field

\section{TABLE I}

The coercivity and anisotropy field for all discussed hysteresis.

\begin{tabular}{c|c|c|c}
\hline \hline Type & $\begin{array}{c}\text { Volume } \\
\text { of hard } \\
\text { area [\%] }\end{array}$ & $\begin{array}{c}\text { Coercivity } \\
\text { of average } \\
\text { hyst. [T] }\end{array}$ & $\begin{array}{c}\text { Anisotropy } \\
\text { field [T] }\end{array}$ \\
\hline S-W parcile & $30-70$ & 8.8 & 17 \\
\hline Hard shpere & 30 & 1.35 & 1.8 \\
in soft & 50 & 3.4 & 3.8 \\
ferromagnetic & 70 & 4.8 & 6 \\
\hline Perlin & 30 & 1.35 & 2.75 \\
noise & 50 & 3.9 & 7.3 \\
geometry & 70 & 6.85 & 12.8
\end{tabular}

increases from $6 \mathrm{~T}$ for the spherical particle up to $12.8 \mathrm{~T}$ for the system with Perlin noise geometry. Moreover, the coercivity of average hysteresis for regular and irregular system is the same in case of 30 vol.\%, but rises from $3.4 \mathrm{~T}$ to $3.9 \mathrm{~T}$ and from $4.8 \mathrm{~T}$ up to $6.85 \mathrm{~T}$ for $50 \%$ and $70 \mathrm{vol} . \%$, respectively. The comparison of all average hysteresis loops was presented in Figure 5. Moreover, the coercivity as well as anisotropy field are summarized in Table I.

\section{Conclusions}

The main conclusions of this work can be summarized as follows:

1. The applied procedure is a good method for simulation of the magnetization processes of the investigated systems.

2. In comparison to a typical $\mathrm{S}-\mathrm{W}$ particle, the ferromagnetic matrix leads to high decrease of the anisotropy field, in our case (50 vol. \%) from $17 \mathrm{~T}$ to $3.8 \mathrm{~T}$ and $7.3 \mathrm{~T}$ for spherical particle and irregular system, respectively.

3. In comparison to spherical particle, irregular geometry of the magnetic phases causes the observed increase of coercivity, especially in case of the systems with high $(>50 \%)$ volume fraction of hard magnetic phase.

4. The Perlin noise provides a good approximation of magnetic composite structure and can be used in simulation of such materials.

\section{Acknowledgement}

This work was supported by the Polish National Science Center by the grant 2015/19/B/ST8/02636.

\section{References}

[1] K.H.J. Buschow , F.R. de Boer, Physics of magnetism and magnetic materials, Kluwer Academic Publishers, 2004.

[2] J.M.D. Coey, Magnetism and Magnetic Materials, Cambridge University Press, 2010.

[3] F.E. Luborsky, J. Appl. Phys. 37, 1091 (1966).

[4] A. Chrobak, G. Ziołkowski, N. Randrianantoandro, J. Klimontko, G. Haneczok, J. Alloys Comp. 537, 154 (2012).

[5] A. Chrobak, G. Ziółkowski, N. Randrianantoandro, J. Alloys Comp. 583, 48 (2014)-54.

[6] A. Chrobak, G. Ziółkowski, N. Randrianantoandro, J. Klimontko, D. Chrobak, K. Prusik, J. Rak, Acta Materialia 98, 318 (2015) 318.

[7] G. Ziółkowski, A. Chrobak, Acta Phys. Pol. A 127, 597 (2015).

[8] K. Perlin, SIGGRAPH Comput. Graph. 19, 287 (1985). 